\title{
Instantaneous Flow Structures in a Reacting Gas Turbine Combustor
}

\author{
Sulabh K. Dhanuka* and James F. Driscoll ${ }^{\dagger}$ \\ Department of Aerospace Engineering, University of Michigan, Ann Arbor, MI 48105, USA \\ Hukam C. Mongia ${ }^{\ddagger}$ \\ GE Aircraft Engines, Cincinnati, $\mathrm{OH}$ 45215, USA
}

\begin{abstract}
While many researchers have studied swirl-stabilized and other model gas turbine combustors, very few have investigated the flow field and combustion within a gas turbine combustor operated with realistic Jet-A fuel at elevated pressures and inlet temperatures. In this paper we present results from an investigation into the flow field and flame locations within a unique combustor furnished with an injector designed by GE Aircraft Engines. The combustor provides high optical access which has allowed the use of Particle Imaging Velocimetry to probe both instantaneous vortex and recirculation zone structures in the flow and obtain ensemble averaged profiles of pertinent turbulence quantities. Both non-reacting and reacting conditions were studied to assess the effects of a flame on the flow within the combustor. All reacting conditions were run with liquid Jet $\mathrm{A}$ as fuel. This too provides a more realistic configuration for it accurately captures the effects of droplet/spray combustion along with the correct heat release from a heavy hydrocarbon fuel. The location of the flame front was imaged using Planar Laser Induced Fluorescence (PLIF) of formaldehyde. This study is one of the first to apply PLIF to a gas turbine combustor running liquid Jet A. Important flow features present in the combustor have been identified and key differences between the reacting and non-reacting flow fields have been noted. With information about both the flow and flame front, structures that have not been highlighted in the past are shown to be important in the operation of the combustor. These results have indicated that the time-averaged flow field masks some very important details of the flow within a gas turbine combustor.
\end{abstract}

\section{Introduction}

Lean Premixed Prevaporized (LPP) combustors offer one of the best methods to meet the goals of reduced NOx emissions. By their very nature however, LPP combustors are prone combustion driven oscillations and unsteadiness, and these instabilities severely limit the operating envelope of such combustors. ${ }^{1}$ The design of stable combustors therefore is an important goal for industry and the combustion community. Swirling flows have been used in a variety of applications to provide the enhanced mixing and shortened residence times that are necessary for a stable flame and low emissions. ${ }^{2-4}$ The flow field and other characteristics of swirl combustors have been studied by other researchers, ${ }^{5-10}$ but these studies have yielded only mean velocity results. The use of Particle Image Velocimetry (PIV) however allows the full instantaneous flow field to be measured and flow structures to be identified. ${ }^{11}$ Past studies of gas turbine combustors have also been limited to atmospheric pressures and gaseous fuels such as methane or natural gas. It is believed that the use of liquid Jet-A fuel at elevated pressures is necessary to accurately capture the physics of a gas turbine combustor. PLIF of formaldehyde was used for the first time in a challenging Jet-A fuel environment to image the flame fronts and flame locations. Standard chemiluminescence imaging used in previous studies is unacceptable because it is a line-of-sight spatial average.

*Research Assistant, AIAA Student Member.

†Professor, Fellow AIAA

¥Manager of Combustion Technologies, AIAA 
For this study, high flowrates (up to $0.38 \mathrm{~kg} / \mathrm{s}$ ) of air at high temperatures (up to $500 \mathrm{~K}$ ) and at elevated pressures (up to 4.5 bar) was supplied to the combustor. This yielded a highly turbulent flow field with a number of pertinent features. A marked difference was observed between the non-reacting and reacting cases. A large recirculation zone exists in the non-reacting case. In the reacting case, this primary recirculation zone is reduced to a much smaller zone near the centerline of the combustor. Essentially, the heat release from the pilot flame causes a rapid expansion and subsequent acceleration of the hot product gases. This therefore reduces the size of the recirculation caused by vortex breakdown behind the injector. This is a key result that suggests important differences between non-reacting and reacting conditions and indicates that non-reacting studies such as those done in a water model or even in gas would lead to erroneous results when extended to a combustor in operation. PIV data indicates the existence of many distinct flow structures in the combustor. These structures are absent from the mean results and help to illustrate the shortcomings of any time-averaged measurement or simulation. This also applies to single point measurements such as laser doppler velocimetry (LDV) where instantaneous field measurements are not available.

\section{I.A. Background}

The GE Twin Annular Premixing Swirler (TAPS) was described by Mongia. ${ }^{12}$ It was designed to reduce the overall $\mathrm{NO}_{x}$ emissions while still providing stable flame conditions. A central pilot operates as a diffusion flame for starting and low power operation and to help stabilize the main flame. ${ }^{12,13}$ The main flame, which is located in an annulus around the pilot, operates in a premixed or partially premixed fashion in order to achieve low $\mathrm{NO}_{x}$ levels. The goal of this work is two-fold. The first is to provide a high quality data set to modelers who wish to model a complicated yet important combustor such as the GE TAPS combustor. Groups have begun to model the combustor and others similar to $i t^{14}$ and data is needed to validate the results. The second is to understand the flow field within such a combustor and better explain the mechanisms by which the low $\mathrm{NO}_{x}$ levels are achieved and how the pilot stabilizes the main flame. It is hoped that the insights gained from such experiments will lead the development of unsteady LES models that will be able to better simulate realistic combustors.

\section{Experimental Setup}

All experiments were conducted in the University of Michigan/GE TAPS Combustor Facility. A TAPS injector was provided by GE Aircraft Engines and mounted in a $20 \mathrm{~cm}$ diameter pressure vessel as shown in Fig. 1. Air from external compressors was heated using a $250 \mathrm{~kW}$ electrical heater and metered via a custom choked flow orifice. The flow passes through flow conditioning sections prior to entering the combustor. The air then enters either the TAPS injector or passes around the injector to enter downstream through cooling holes. Within the TAPS injector, the majority of the air passes through the main swirler while the remainder enters the combustor through two co-rotating pilot swirlers. A valve downstream of the combustor and prior to the exhaust allows the combustor pressure to be varied. A central atomizer injects the pilot fuel while the main fuel is injected by discrete transverse jets placed around the periphery of the TAPS injector. The fuel used was liquid Jet-A. The main fuel jet is injected into an air cross flow. This provides a high degree of mixing and gives this combustor its partially premixed to premixed character. Optical access is available on three sides of the combustor via 5" diameter fused silica sight windows on the outer pressure vessel and rectangular fused silica windows on the inner, main flametube. The inner flametube is nominally cylindrical

but has been fitted with three planar windows on three sides. Two sets of windows were required to isolate the main combustor from the outer baffle. This setup allows good optical access near the injector.

\section{II.A. Laser Diagnostics}

PIV was used to map out the shear layers, recirculation zones, turbulence intensities, and the effect of the flames on the velocity field. The bulk air stream was seeded with nominally 0.5 micrometer diameter alumina particles which adequately tracked the flow. This fact can be verified by computing the Stokes number of the flow. The particle relaxation time for the seed is $\tau_{p} \approx 2.85 \mu \mathrm{m}$. The flow time scale was computed as $\tau_{f} \approx 60 \mu \mathrm{s}$ by assuming a worst case gradient with the velocity being the highest values measured $(40 \mathrm{~m} / \mathrm{s})$ and the length being the interrogation window size $(2.34 \mathrm{~mm})$. With this, the worst case Stokes number is $S t \approx 5 \times 10^{-3}$, which is much less than the criterion of $S t<0.5$ suggested by ${ }^{15}$ and. ${ }^{16}$ Excessive flame luminosity due to the use of Jet-A was a considerable problem faced in this study. To overcome this, an 


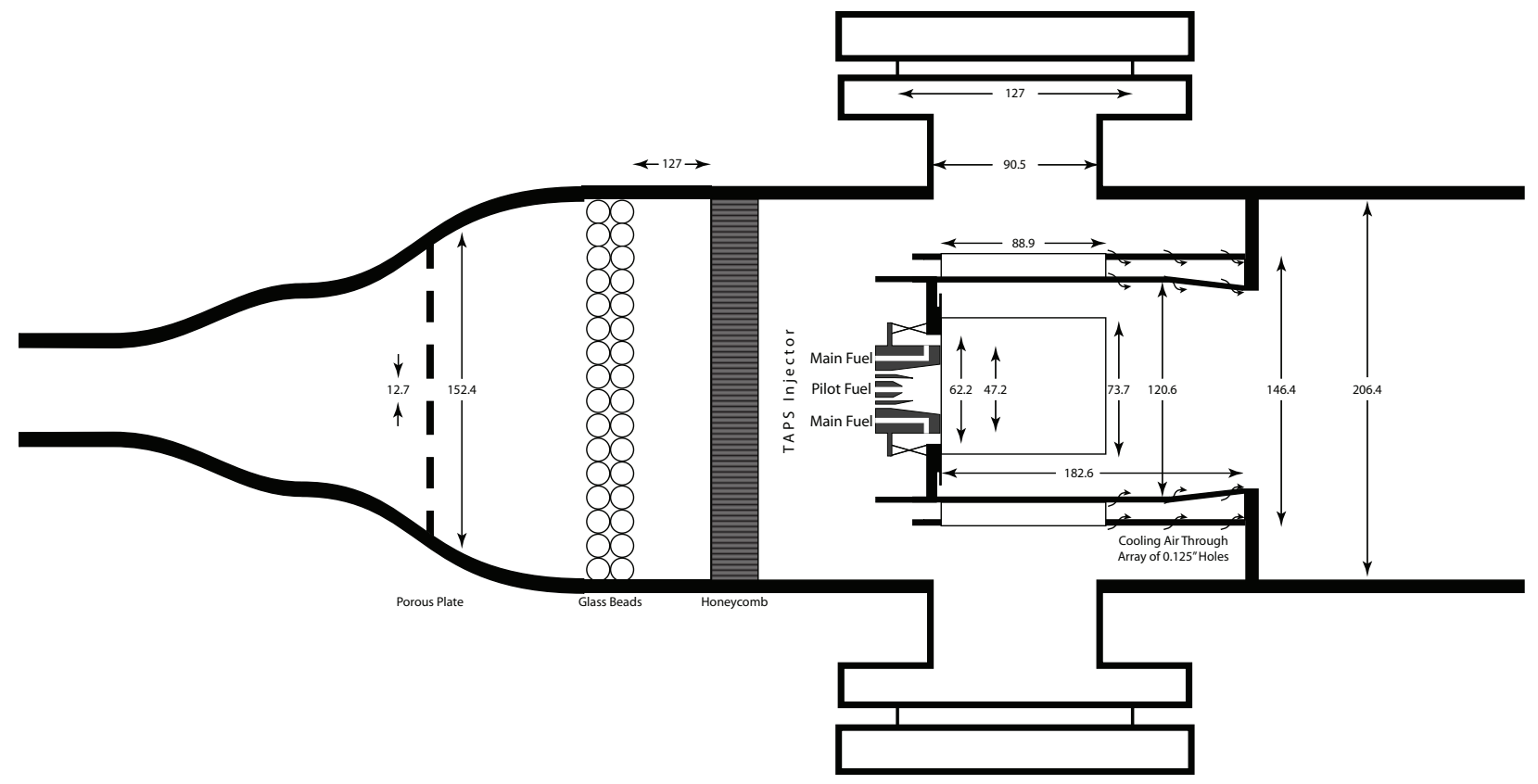

Figure 1. Schematic of the UofM/GE TAPS combustor shown to scale (except for the flow conditioning section). The flow enters from a two inch pipe through a diffuser housing the flow conditioning elements in a $600 \mathrm{~mm}$ long section. The pilot swirlers are not drawn for clarity and the inner rectangle reflects the inner windows. All dimensions are in mm.

interference filter at $532 \mathrm{~nm}$ with a FWHM of $2 \mathrm{~nm}$ and a mechanical shutter were fitted to the camera lens. The final interrogation window size of $64 \times 64$ pixels yielded a spatial resolution of $2.34 \mathrm{~mm}$.

The flame was imaged using PLIF of formaldehyde $\left(\mathrm{CH}_{2} \mathrm{O}\right)$ which is an important combustion intermediate that is formed on the reactant side and quickly destroyed at the flame front. Its chemistry and spectral characteristics have been extensively studied ${ }^{17-20}$ and has since been applied in a variety of flames. ${ }^{21-23}$ PLIF of $\mathrm{CH}_{2} \mathrm{O}$ offers two advantages that make it the best choice for imaging the flame in Jet-A gas turbine environment. First, it has been shown that while signal from excited soot and PAH's can be saturated, emissions from formaldehyde do not saturate. ${ }^{20,21}$ This allows the flame boundary to be accurately imaged even in the presence of soot and other PAH's by using a sufficient power laser. Secondly, $\mathrm{CH}_{2} \mathrm{O}$ can be excited at $355 \mathrm{~nm}$, the third fundamental of an Nd:YAG laser, which provides the high power needed. The third fundamental of a Spectra Physics (GCR-250) Nd:YAG laser at $355 \mathrm{~nm}$ was used with a pulse energy of $150 \mathrm{~mJ}$. The laser was passed through sheet forming optics to form a $70 \mathrm{~mm}$ x $0.4 \mathrm{~mm}$ sheet. The resulting fluorescence was imaged with an Andor ICCD (iStar 734) fitted with Schott glass GG-385 and BG-3 filters. The camera was also fitted with a mechanical shutter to block out excessive flame luminosity. Even though short gate times (100 ns) were used, there was considerable background noise from flame luminosity that "leaked" through the intensifier. The $1 \mathrm{k}$ x $1 \mathrm{k}$ CCD was binned $2 \mathrm{x} 2$ to yield a spatial resolution of $0.15 \mathrm{~mm}$. This study marks the first time that PLIF has been utilized to mark the reaction zone in a Jet-A fuelled gas turbine combustor.

\section{Mean Flow Results}

Results from one reacting and one non-reacting condition will be presented here. For the purposes of this study, only the effect of the pilot flame was studied and no fuel was injected through the main fuel injectors. The specifics of the non-reacting and reacting conditions are given in Table 1.

\section{III.A. Recirculation Zones}

The mean velocity fields for both the non-reacting and reacting cases are shown overlayed on the contours of average axial velocity in Fig. 2. Since the geometry and flow are nominally axisymmetric, only one half of the combustor is shown. The TAPS geometry (shown as part of the combustor in Fig. 1) is shown for 


\begin{tabular}{|c|c|c|c|c|}
\hline Case & $\begin{array}{c}\dot{m}_{\text {air }} \\
(\mathrm{kg} / \mathrm{s})\end{array}$ & $\begin{array}{c}T_{3} \\
(\mathrm{~K})\end{array}$ & $\begin{array}{c}p_{3} \\
(\mathrm{bar})\end{array}$ & $\begin{array}{c}\dot{m}_{\text {fuel }} \\
(\mathrm{g} / \mathrm{s})\end{array}$ \\
\hline \hline Non Reacting & 0.377 & 503 & 4.54 & 0 \\
Reacting & 0.379 & 498 & 4.54 & 2.79 \\
\hline
\end{tabular}

Table 1. Details of the two cases for which data is presented. The mass flowrate of air ( $\left.\dot{m}_{a i r}\right)$ is the total flowrate of air that goes through both swirl annuluses and cooling holes.

reference in solid black at the left of the velocity results. The origin of the cartesian coordinates used in the results was defined as being on the dump plane and center of the injector for $x$ and $y$ respectively. The pilot flame is at the center, surrounded by the pilot two annuluses. The main swirl annulus is located between $y=23.6 \mathrm{~mm}$ and $y=31.1 \mathrm{~mm}$. The boundary of the recirculation zone is marked by the boundary of negative axial velocities and shown by the black lines. The thick red line in the reacting flow field indicates the average flame contour obtained from the $\mathrm{CH}_{2} \mathrm{O}$ PLIF results (§III.C).

Immediately apparent is the drastic change in the primary recirculation zone (PRZ) between the reacting and non-reacting cases. This recirculation zone has been well documented in all similar swirl combustors and is created through vortex breakdown. In the non-reacting case, the PRZ encompasses almost the entire field of view. This PRZ is considerably reduced in the reacting case and forms a small zone that is pushed both downstream and axially inward. The heat release from the pilot flame causes rapid expansion of the gas which reduces the size of the PRZ. The acceleration of the flow also appears to break the PRZ into two separate zones as seen from the smaller zone of negative velocity in the upper right corner. While changes in the size and shape of the PRZ due to heat release have been observed in other studies, such a drastic reduction in size and shape have not. Interestingly, the flow on the centerline is positive and has broken the PRZ into the smaller bubble shown. The TAPS injector has unique a pilot swirl annulus that allows air to be issued close to the centerline but the centerline flow cannot be accredited to that since a similar feature does not exist in the non-reacting case. The flow issuing from the pilot annulus also issues at a higher velocity than in the non-reacting case while the main flow is largely unaffected.

A smaller recirculation zone is also seen around $x, y=(7,24) \mathrm{mm}$ near the lip of the injector. This lip recirculation zone (LRZ) is caused by the wake of the injector upstream of the point that the pilot flow merges with the main flow. This zone is a unique feature of the TAPS injector (due to the twin annular design) that has been shown to play a prominent role in the stability of the combustor. ${ }^{24}$

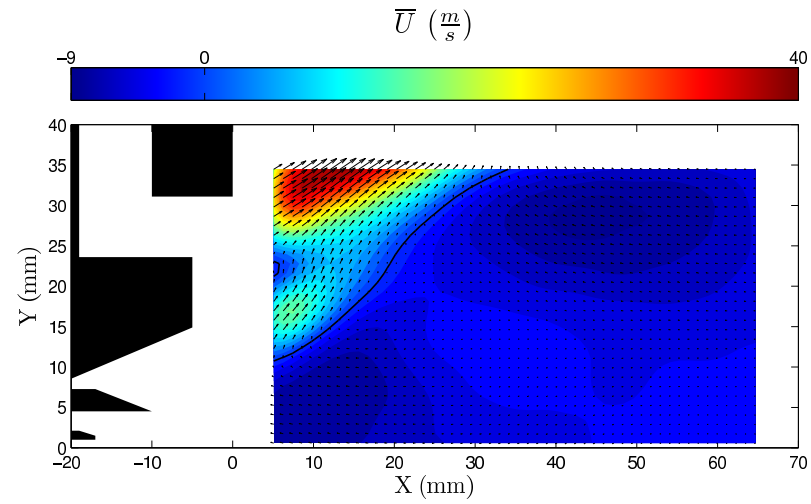

(a) Non-Reacting

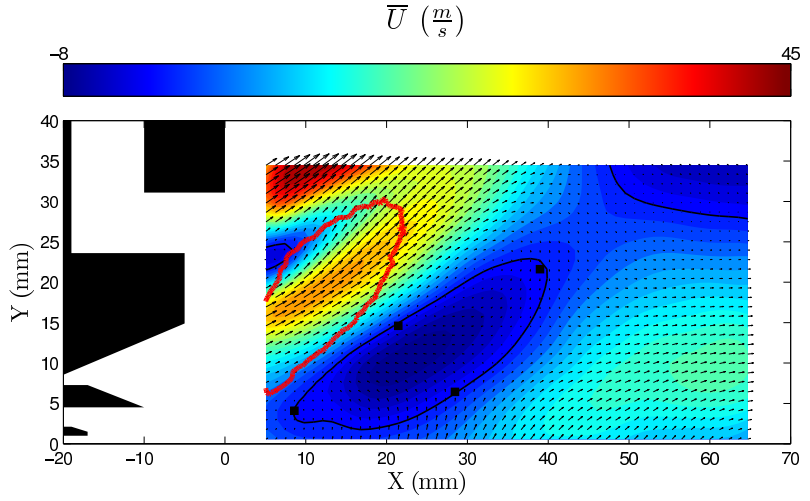

(b) Reacting

Figure 2. Contour plots of the average axial velocities for the non-reacting (left) and reacting (right) flow fields. The average flame contour from $\mathrm{CH}_{2} \mathrm{O}$ PLIF is overlayed in red. The boundary of the regions of negative axial velocity is marked by the black line in both images. The solid square markers around the PRZ in the reacting case denote the locations used for the results shown in Fig. 11. 


\section{III.B. Pertinent Mean Quantities}

Contour plots of several pertinent mean quantities help illustrate a number of other key differences caused by the heat release from the flame. Shown in Fig. 3 and 5 are contours of average radial velocity, vorticity, turbulence intensity, and shear strain rate for the non-reacting and reacting cases respectively. Profiles of the quantities shown in the contour plots also help illustrate the ideas discussed above and provide for easy comparison with models or other results of similar combustors. Profiles for the non-reacting case are shown in Fig. 4 and those for the reacting case are shown in Fig. 6.

The vorticity is the out-of-plane vorticity $\left(\omega_{z}\right)$ calculated from the two measured components of velocity as $\omega_{z}=\frac{\partial v}{\partial x}-\frac{\partial u}{\partial y}$. The turbulence intensity is calculated as $q=\sqrt{u^{\prime 2}+v^{\prime 2}} / 2$ ). The shear strain rate is another very important quantity in turbulence chemistry interactions and is calculated as $\varepsilon_{x y}=\frac{\partial u}{\partial y}+\frac{\partial v}{\partial x}$. The contours for the non-reacting case indicate a fairly steady recirculation zone devoid of any features. The PRZ has near trivial values of vorticity and shear strain rate. This fact is also seen from the profiles in Fig. 4 where by about $x=25 \mathrm{~mm}$ the vorticity is almost zero. Even the turbulence intensity, though not zero, displays a near constant value across the combustor. As would be expected, the high vorticity and strain rate values are in the shear layer near the edge of the PRZ. The boundaries of the PRZ and LRZ are shown again in the contour plot of vorticity where the vorticity contours indicate the vortices present near the edges. The profile of vorticity shows four distinct peaks of vorticity at $x=5 \mathrm{~mm}$ that either move radially outward or merge into one peak as the PRZ grows in the radial dimension. The highest values of turbulence intensity are in the flow issuing from the two annuluses as would be expected for a flow at these Reynolds numbers.
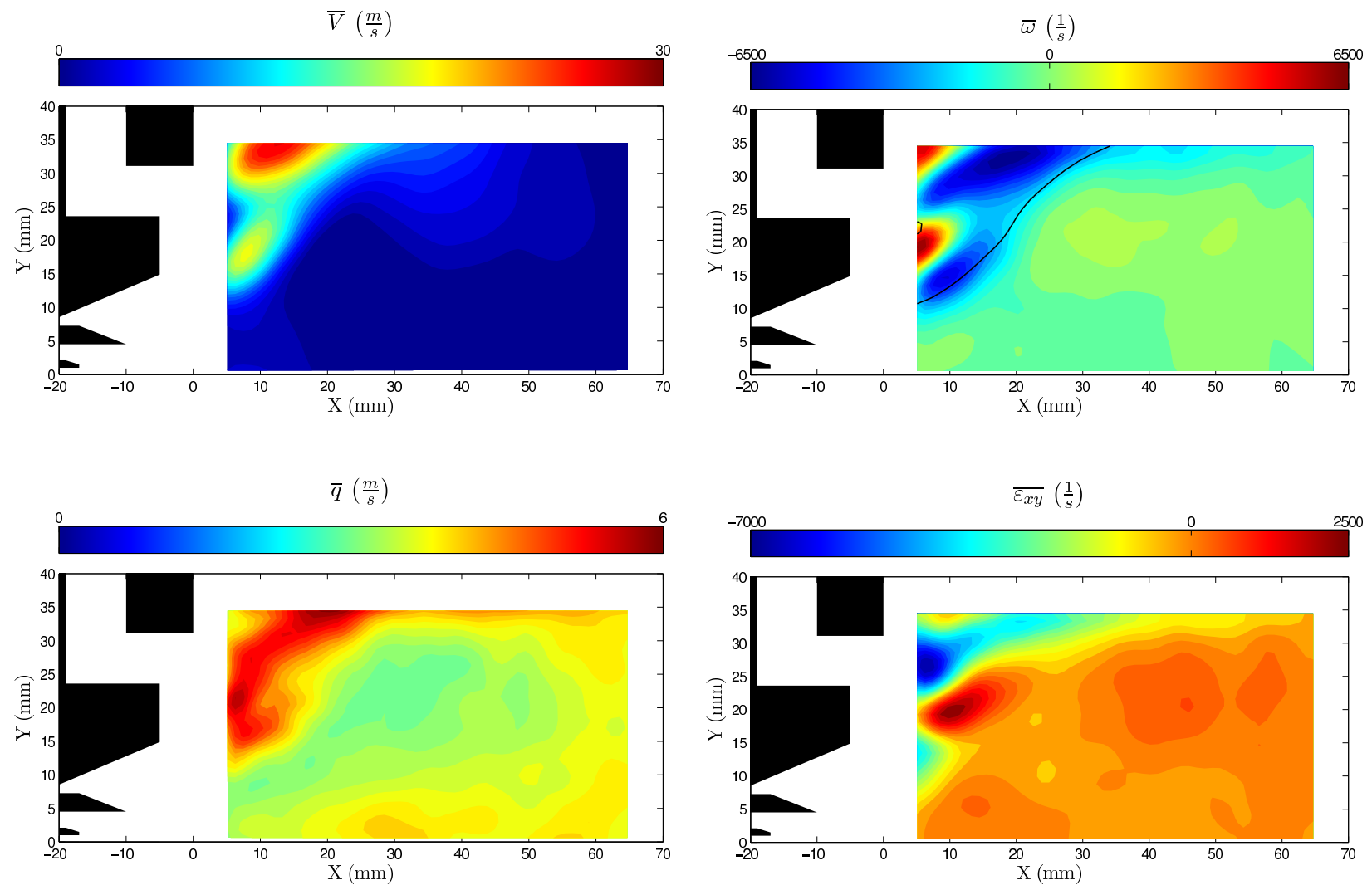

Figure 3. Contour plots of select averaged quantities for the non-reacting flow field.

The mean quantities shown in Fig. 5 and Fig. 6 support the result from Fig. 2 that the existence of the pilot flame leads to significant differences in the flow field. The heat release from the flame causes the increased values of turbulence intensity along the centerline and near the pilot flame cone. Along the centerline the turbulence intensity is nearly constant and extends through the length of the combustor as hot product gases accelerate out of the combustor. Interestingly, the turbulence levels of the flow issuing from the main annulus are lower than in the non-reacting case, even though the axial and radial velocities are very 

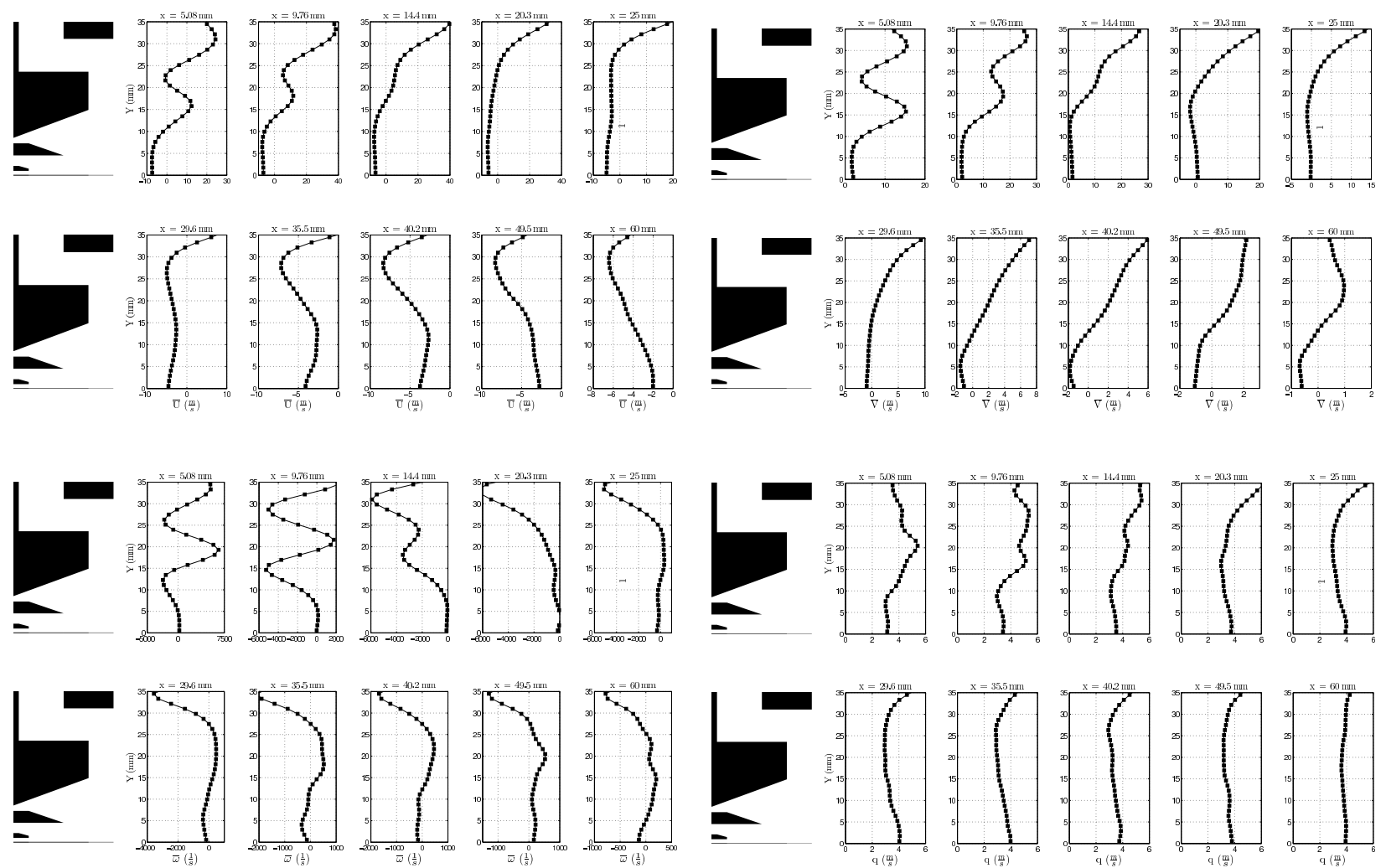

Figure 4. Profiles of the non-reacting flow field shown at select downstream locations.

$\bar{V}\left(\frac{m}{s}\right)$

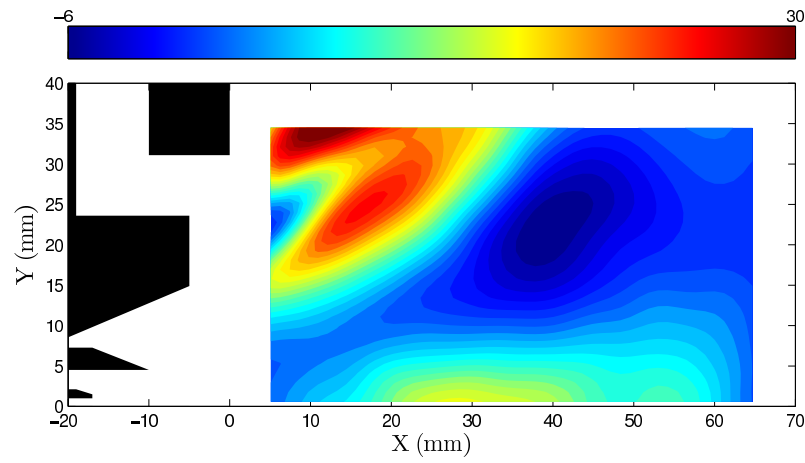

$\bar{q}\left(\frac{m}{s}\right)$

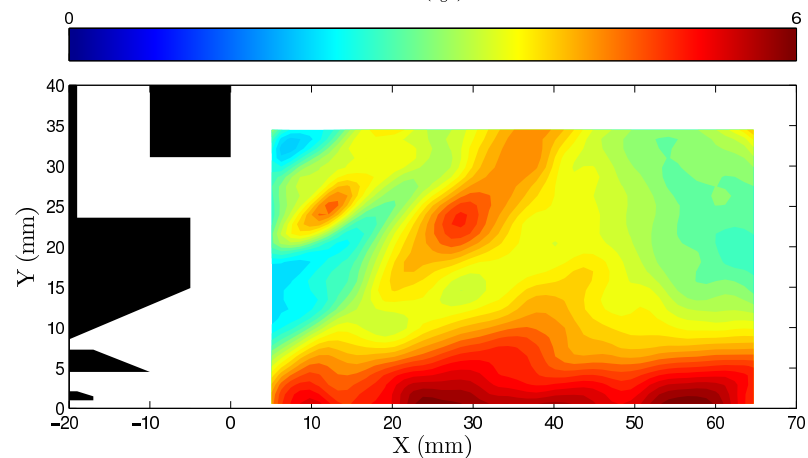

$\bar{\omega}\left(\frac{1}{s}\right)$

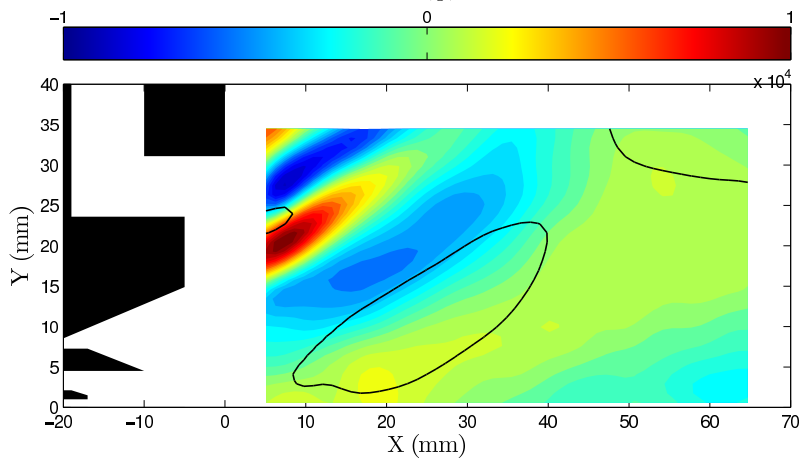

$\overline{\varepsilon_{x y}}\left(\frac{1}{s}\right)$

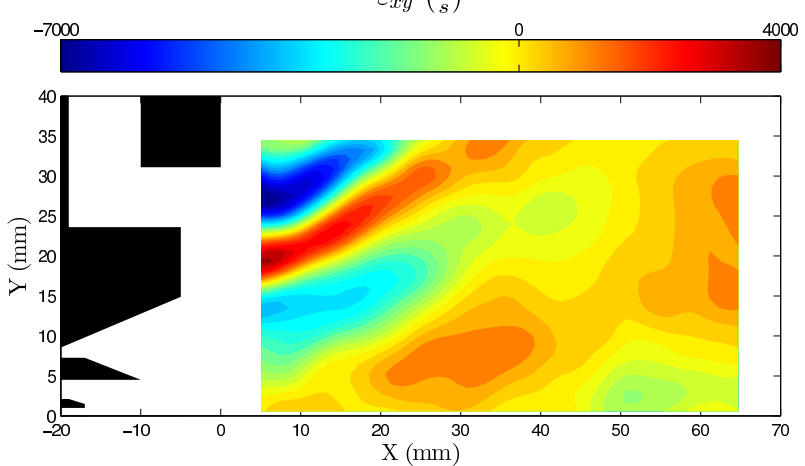

Figure 5. Contour plots of select averaged quantities for the reacting flow field. The boundary of the regions of negative axial velocity is marked by a black line in the image of average vorticity. 

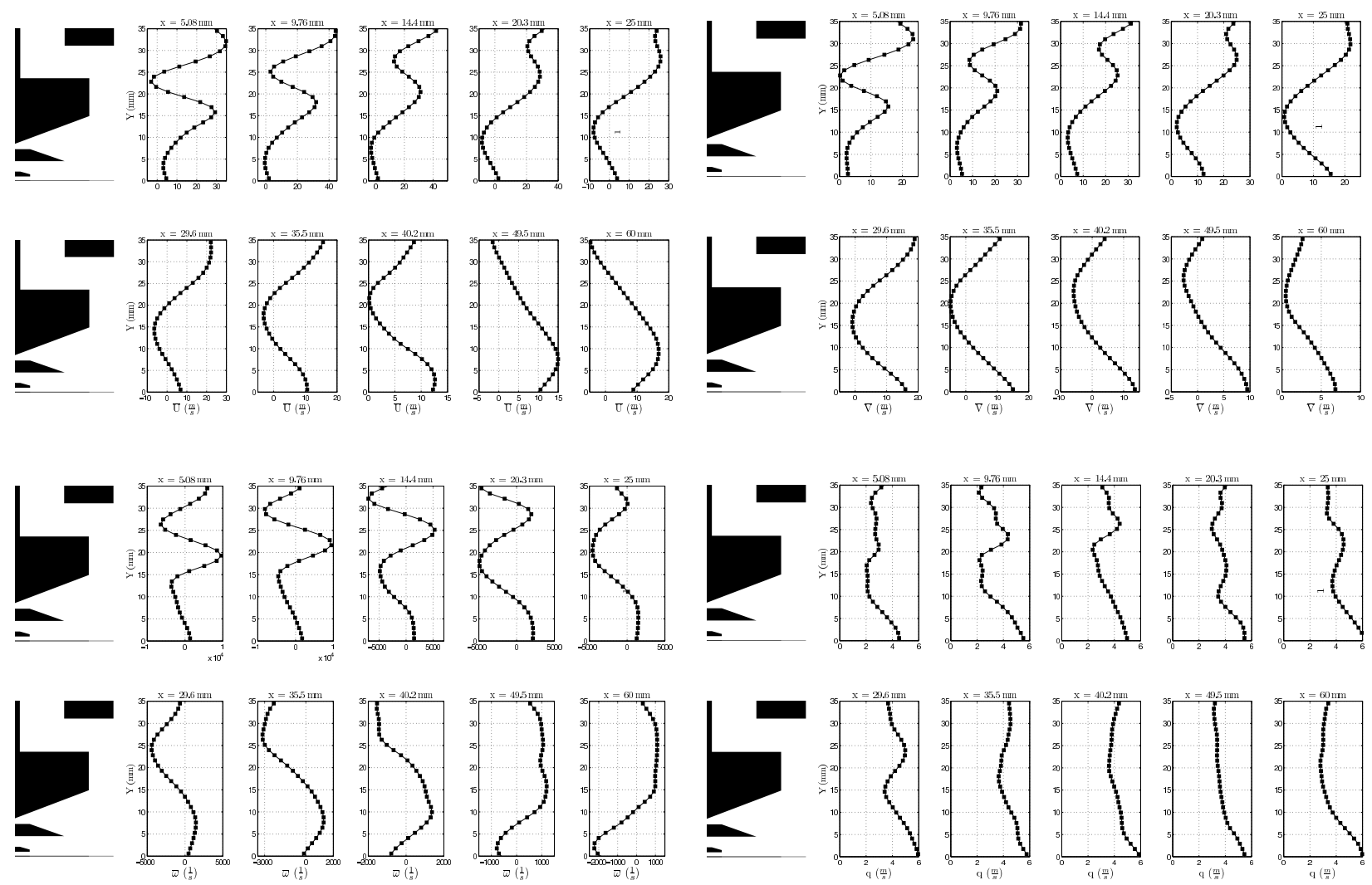

Figure 6. Profiles of the reacting flow field shown at select downstream locations.

similar in both cases. Since the flow from the main annulus issues into the pilot flame (see Fig. 2b), the heat release upstream reduces the turbulence levels going into the flame. This suggests that the pilot flame aids in the stable combustion of a main flame by not only providing hot products but by reducing the turbulence intensity of the main flow. Similar to the non-reacting case, the highest values of both gradient quantities, the vorticity and strain rate, are concentrated in the shear layers surrounding the recirculation zones. The high strain rates near the flame suggest that the pilot flame is subject to a high degree of flame stretch and wrinkling. Since high strain rates also lead to high local anisotropy, models with inherent assumptions of isotropy will likely fare poorly in these regions. While it is obvious that the heat release from the pilot causes important changes in the flow field downstream of the flame cone, the changes in the flow upstream are only nominal. This is best illustrated by comparing the profiles of the mean quantities at $x=5 \mathrm{~mm}$ for both the reacting and non-reacting cases. Apart from the difference in the turbulence intensity mentioned above, the basic features and magnitudes are well preserved upstream of the flame.

\section{III.C. PLIF Results}

PLIF of $\mathrm{CH}_{2} \mathrm{O}$ was used to image the location of the pilot flame which provides an accurate cross-section of the flame. The PLIF images were acquired at a lower pilot flowrate than the flowrate used in the velocity measurements but studies by the authors have shown that while the flame length is altered, there is no change in shape or cone angle with fuel flowrate. Also, the presence of high amounts of soot and PAH's at elevated pressures necessitated the acquisition of the PLIF signal at a combustor pressure of 1 bar. Again, studies of the flame at higher pressures do not suggest that there is a remarkable effect of combustor pressure on the shape or location of the flame. The average PLIF signal is shown in Fig. 7a and shows the formaldehyde concentrations in a cone issuing from the pilot injector at approximately $45^{\circ}$. The outer (towards the main annulus) edge of the formaldehyde concentrations near the base have a much higher radial gradient than the inner (towards the centerline) edge. This is because the LRZ is right against the outer edge which constrains the flame. In the inner edge the PRZ is further away but still leads to a remarkably straight edge of the inner flame cone on average. The flame brush shown in Fig. $7 \mathrm{~b}$ was obtained by plotting select (chosen 
randomly) flame contours from instantaneous PLIF images. The average flame contour, obtained from the average PLIF image shown in Fig. 7a is overlayed in black over the instantaneous contours (in gray). It is important to note that the average contour is not an average of the instantaneous contours but rather the contour from the average PLIF image. The flame brush shows the highly turbulent nature of the flame with a high degree of flame wrinkling. While the instantaneous flames are highly wrinkled, they do appear to be confined to a small region (of about $2-3 \mathrm{~mm}$ ) around the average flame contour.

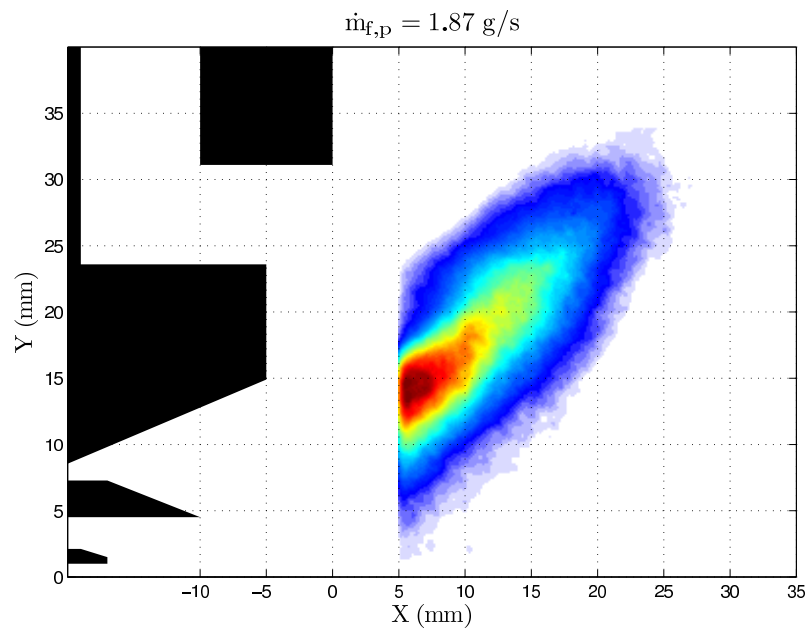

(a) Average PLIF Signal

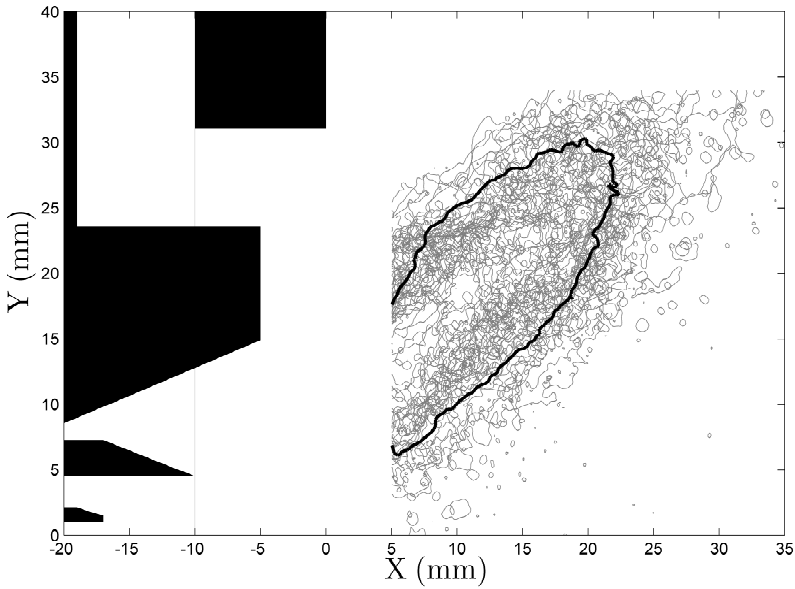

(b) Flame Brush

Figure 7. The average PLIF signal along with selected flame contours taken from instantaneous PLIF images. The average flame contour is overlayed on the flame brush as a thick red line.

\section{Instantaneous Flow Results}

An important finding of this study has been the fact that there is considerable structure in the flow that is masked in the average results shown earlier (§III). Shown in Fig. 8 are four randomly selected vector plots of the reacting case. To better highlight the structures, the mean flow has been subtracted from the instantaneous velocity measurements. In this manner, the average bulk convection velocity has been subtracted which allows for individual features to be visualized independently. The average flame contour is also overlayed on all the vector plots to provide an idea of the location of the pilot flame. It was seen that the instantaneous flame is located within a few millimeters of the average and therefore the average can be used as a good reference for the general location of the flame. It must be stressed though that simultaneous measurements of velocity and flame location are needed for an accurate understanding of turbulence-chemistry interactions. Nevertheless, the location of the average flame contour does provide for some idea of the flow in the vicinity of the flame. Immediately apparent from all four images is the level of detail present in the instantaneous images that is not present in the average. Two important types of flow structures, namely vortices and stagnation points, are observed. The vortices appear as distinct regions of swirling flow where the streamlines spiral into or out of one coordinate. This is best seen for example at $x, y=(15,20) \mathrm{mm}$ in Fig. 8c where a vortex rotating CCW exists in the middle of the flame. Similarly, in Fig. 8d a number of vortices of both positive and negative vorticity exist on either side of the flame contour. These vortices transport hot products and fresh reactants and aid in the stability of the flame. These vortices also lead to an enhanced flame surface area due to their ability to stretch and wrinkle the flame surface. The other type of structure observed was stagnation point or opposed flow. This type of flow as seen for example at $x, y=(12,10) \mathrm{mm}$ in Fig. $8 \mathrm{~b}$ is similar to a classic opposed diffusion flame. Correctly modelling the associated strain rates therefore becomes important in aiding our understanding of local extinction and flame blowout in such complex geometries. Structural details such as these are also seen away from the flame in the PRZ and other regions in all four images. Also, at any instant in time, the flow around the inner edge of the flame is seen to be either issuing into the flame (Fig. 8a) or away from it (Fig. 8c).

The level of detail is also obvious from the vorticity contours of the selected snapshots as shown in Fig. 9. 
The color scale has been set to the same color scale used for the average vorticity plot (Fig. 5) to allow comparison between the average and instantaneous results. The instantaneous vortices are not only higher in number but also in magnitude. In the region in and downstream of the PRZ, the average contours and profiles suggest an almost trivial value of vorticity. The instantaneous images however show that in fact their exist many individual eddies of opposite sign. These eddies convect at the local velocity and therefore over a sufficient number of samples cancel each other out, leading to a trivial result for $\overline{\omega_{z}}$. This fact presents a problem to average based models such as RANS where only the mean quantities are computed. Any such model would erroneously conclude that the flow field has negligible vorticity and structure.

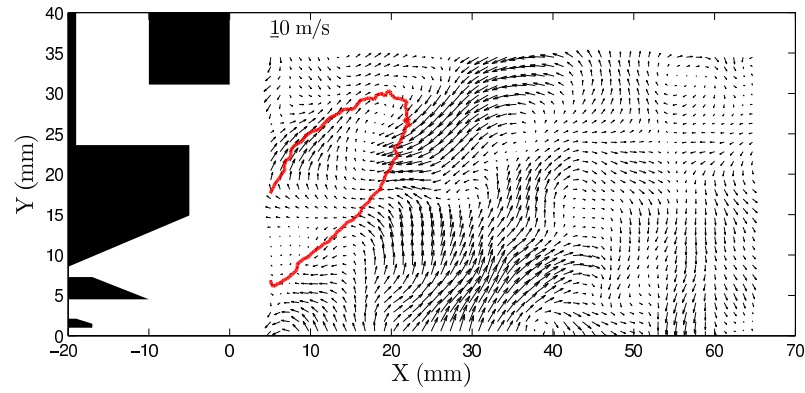

(a)

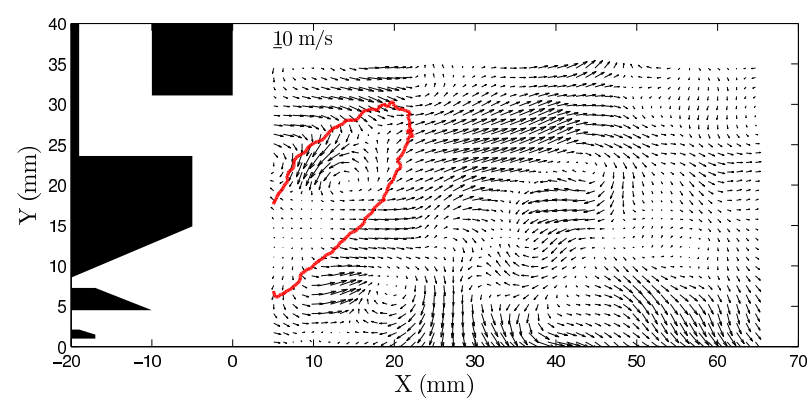

(c)

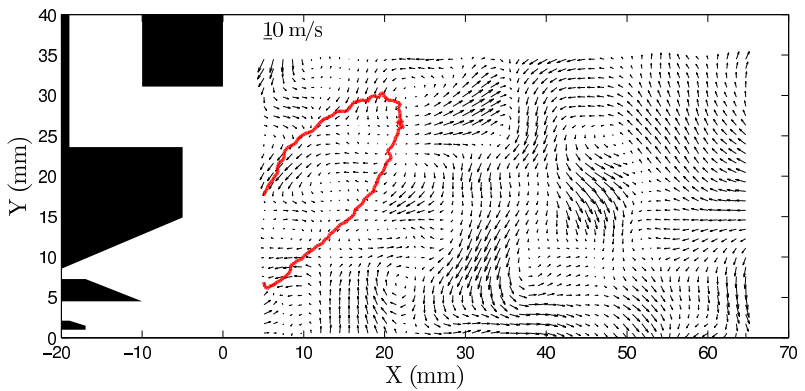

(b)

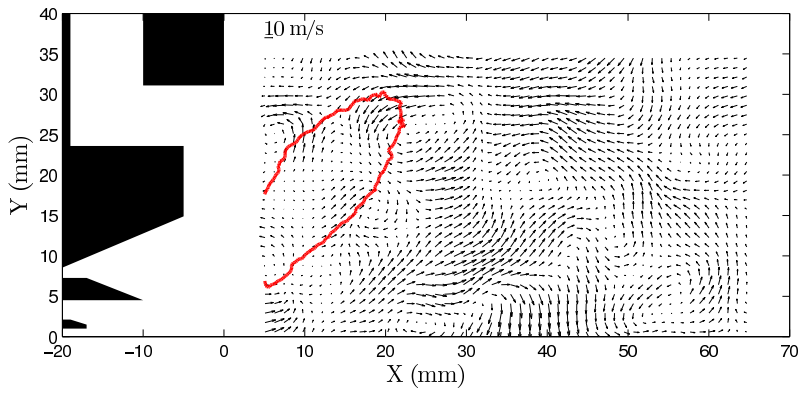

(d)

Figure 8. Randomly selected instantaneous vector plots for the reacting case. The average flame contour is shown as a solid red line in all images.

\section{IV.A. Instantaneous Recirculation Zone}

The above results suggest that the average recirculation zones are also possibly misleading. To assess this, the area of the the recirculation zones was computed by measuring the area of the field of view in which the axial velocities are negative. Since PIV data is naturally discretized by the interrogation windows, this task was accomplished by counting the number of interrogation windows that had $U<0$. This was done for all the instantaneous images and separately for the average image. The PDF of the recirculation zone area is shown in Fig. 10 with the data shown by the solid markers. A Gaussian distribution with the same mean and standard deviation has also been fit to the data. The mean from the instantaneous images is seen to be $619 \mathrm{~mm}^{2}$ while the area computed from the average image is only $456 \mathrm{~mm}^{2}$, which is almost one standard deviation less than the actual mean value. As the PRZ is a critical feature in swirl combustors and is important in the stability of the flame, its area is a vital parameter in the combustor's design. Since the average shape and size of the recirculation zones were obtained by averaging the velocities (and not the areas), this discrepancy can be attributed to the instantaneous values of velocity around the PRZ.

To understand the reason behind the discrepancy in the computed areas, the instantaneous axial velocities were probed at four locations on the border of the average PRZ. These locations were denoted by the solid markers in Fig. 2b. The PDF of the axial velocity at these four locations are shown in Fig. 11 along with their coordinates. As can be seen from the Gaussian fits to the data, a normal distribution appears to be a close fit to the data but naturally does not capture the skewness present in the PDF's. The third 


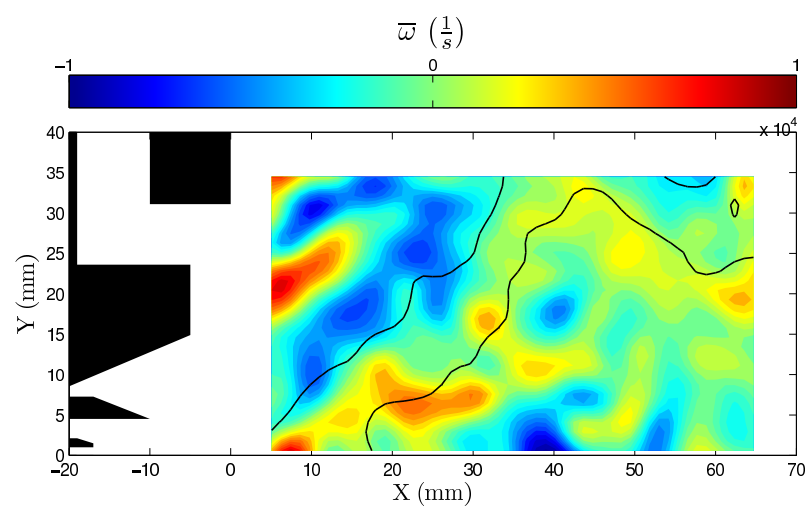

(a)

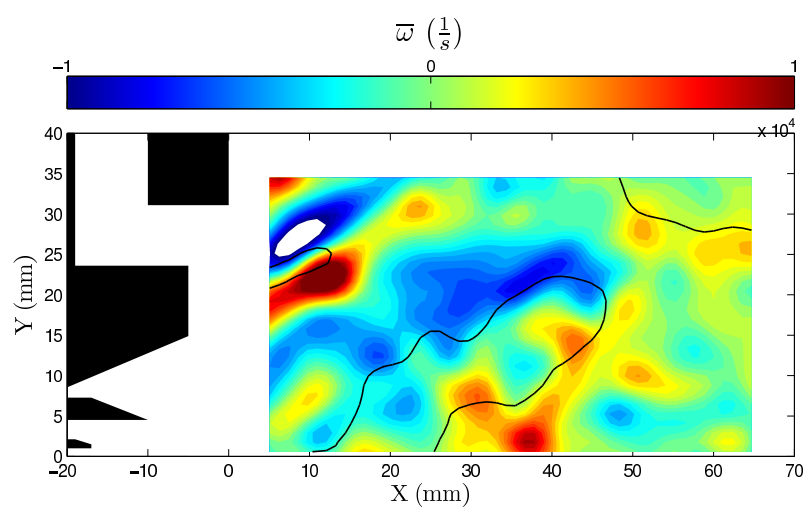

(c)

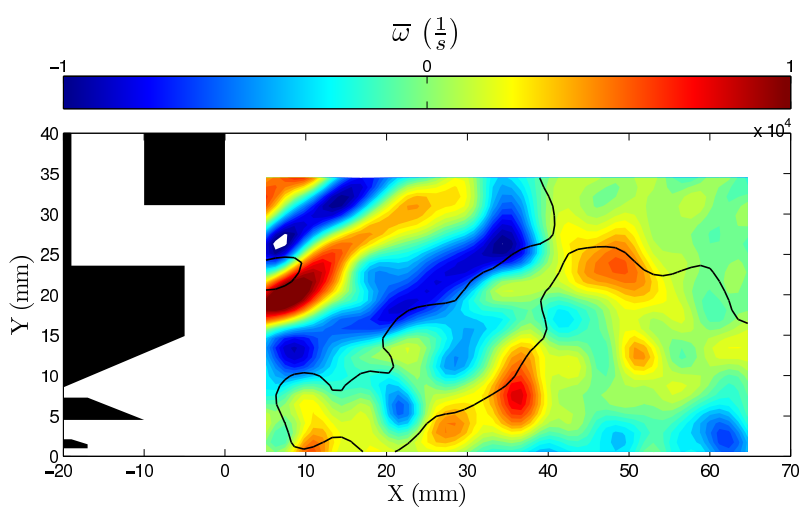

(b)

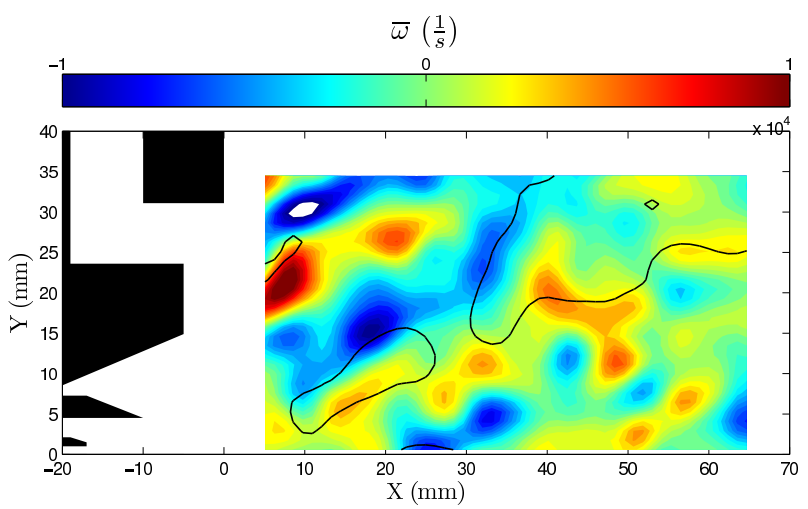

(d)

Figure 9. Contour plot of the vorticity for the select instantaneous images shown in Fig. 8 . The color scale has been set to the same color scale as used for the contour plot of average vorticity (Fig. 5) for ease of comparison. The RZ boundaries are denoted by the black line(s).

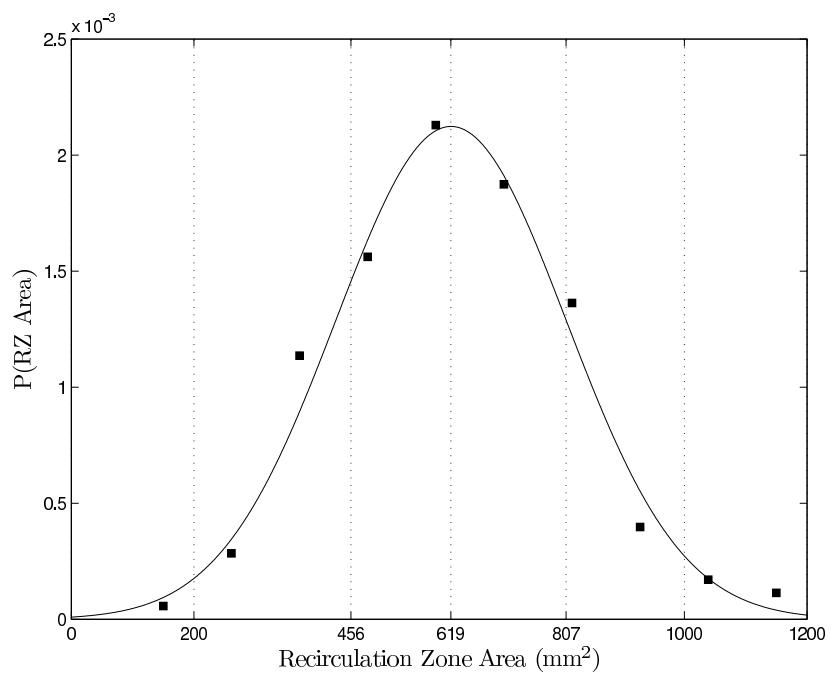

Figure 10. PDF of the area of the recirculation zone(s) in $x \in(5,66), y \in(-5,36)$ shown by the solid markers. The mean of the instantaneous areas is $619 \mathrm{~mm}^{2}$ while the recirculation zone area of the average flow field is $456 \mathrm{~mm}$. The line is a normal (Gaussian) distribution with the same mean and standard deviation $\left(188 \mathrm{~mm}^{2}\right)$ as the data. 
moment, or skewness, of the PDF at each location is listed below the corresponding PDF and is positive at all four representative locations. This means that there are instances of high positive axial velocity (without a corresponding instance of high negative axial velocity) that skew the average results towards a smaller recirculation zone than actually present. This result further illustrates that models relying upon averaged quantities will fail at accurately representing the physics of a swirl combustor. Furthermore, these results also suggest that Gaussian statistics may not be the best choice for describing certain flow features.
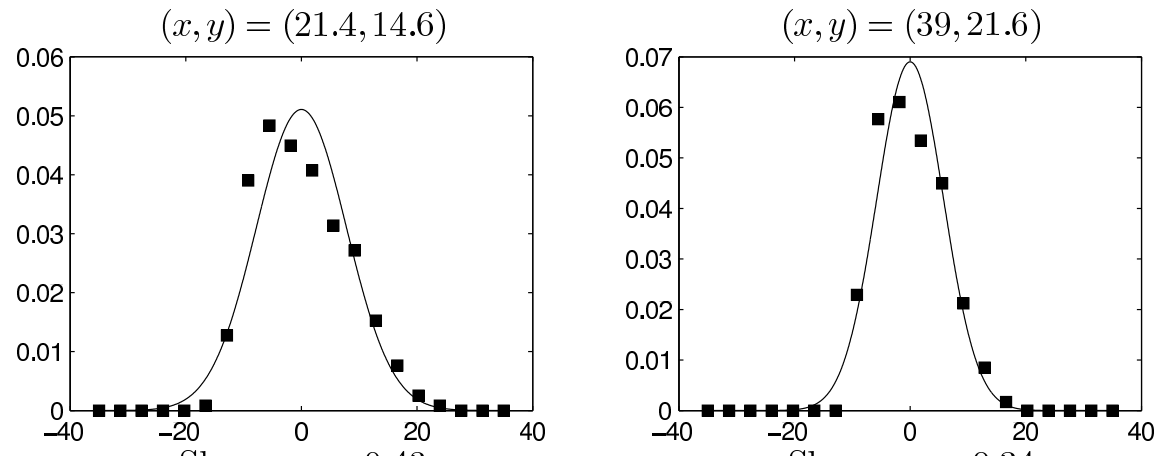

Skewness $=0.43$

Skewness $=0.34$

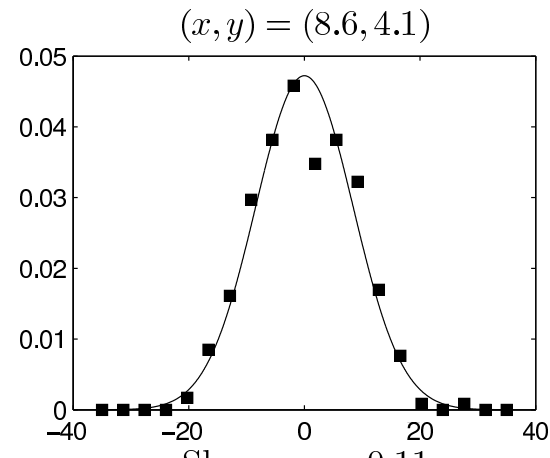

Skewness $=0.11$

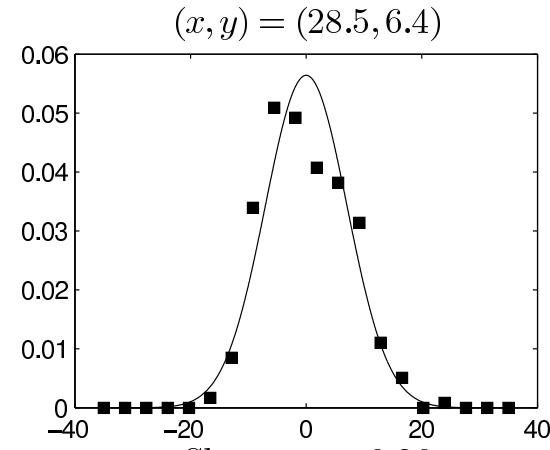

Skewness $=0.26$

Figure 11. PDF of the axial velocity at select locations around the PRZ as marked by the symbols in Fig. $2 \mathrm{~b}$. The lines are Gaussian fits to the data. The third moment (skewness) at each point is given below each plot.

\section{Conclusions}

Advanced laser diagnostics (PIV and PLIF) have been applied for the first time to a Jet-A fuelled gas turbine combustor at elevated pressures and inlet air temperatures. A GE TAPS injector was studied in a high pressure combustor facility at the University of Michigan to obtain a better understanding of the operation of realistic LPP gas turbine combustors.

1. The heat release from the flame drastically changes the flow field within the combustor. The single largest difference is in the size and shape of the primary recirculation zone (PRZ). While the PRZ encompasses almost the entire combustor in the non-reacting case, it is considerably reduced in size in the reacting case. Heat release from the flame causes an expansion of the hot product gases which break the region of negative axial velocities. The lip recirculation zone (LRZ) upstream of the pilot flame is largely unaffected by heat release.

2. The pilot flame exists in a cone upstream of the PRZ and in the shear layer between the flow issuing from the pilot annulus and the PRZ. The highly wrinkled instantaneous flame contours indicate a flame brush that is constrained to a small region near the average flame location.

3. The time-averaged vorticity and strain rate are large in the shear layers but are very small in the bulk of the combustor.

4. The instantaneous flow highlights many distinct flow features that are absent in the time-averaged data. These structures interact with the flame are therefore important to capture. Many individual 
eddies with high values of both positive and negative vorticity exist throughout the combustor. These strong vortices cancel each other out and cause the time-averaged vorticity field to be small.

5. The mean of the areas of the instantaneous recirculation zones is much higher than the area in the average flow. This discrepancy is due to the positive skewness in the PDF's of axial velocity around the PRZ.

6. The results presented in this study have highlighted the importance of utilizing transient codes and models that capture the instantaneous flow field. It is clear that models such as RANS that rely on the mean quantities are unable to accurately simulate the physics of realistic gas turbine combustors.

\section{Acknowledgments}

This research is funded by the General Electric Aircraft Engines University Strategic Alliances (USA) Program. The technical monitor is Dr. Hukam C. Mongia, the GE-USA program manager is Dr. David C. Wisler and the contract number is $14 \mathrm{~F} 35124$.

\section{References}

${ }^{1}$ Lieuwen, T. and McManus, K., "Combustion Dynamics in Lean-Premixed Prevaporized (LPP) Gas Turbines," Journal of Propulsion and Power, Vol. 19, 2003, pp. 721-721.

${ }^{2}$ Beér, J. and Chigier, N., Combustion Aerodynamics, Robert E. Krieger Publishing Company, 2nd ed., 1983.

${ }^{3}$ Syred, N. and Beér, J., "Combustion in Swirling Flows - A Review," Combustion and Flame, Vol. 23, 1974, pp. 143-201.

${ }^{4}$ Weber, R. and Dugue, J., "Combustion Accelerated Swirling Flows in High Confinements," Progress in Energy and Combustion Science, Vol. 18, 1992, pp. 349-367.

${ }^{5}$ Weigand, P., Meier, W., Duan, X. R., Stricker, W., and Aigner, M., "Investigations of Swirl Flames in a Gas Turbine Model Combustor I. Flow Field, Structures, Temperature, and Species Distributions," Combustion and Flame, Vol. 144, No. 1-2, 2006, pp. 205-224.

${ }^{6}$ Janus, B., Dreizler, A., and Janicka, J., "Experimental Study on Stabilization of Lifted Swirl Flames in a Model GT Combustor," Flow, Turbulence and Combustion, Vol. 75, 2005, pp. 293-315.

${ }^{7}$ Schneider, C., Dreizler, A., and Janicka, J., "Fluid Dynamical Analysis of Atmospheric Reacting and Isothermal Swirling Flows," Flow, Turbulence and Combustion, Vol. 74, 2005, pp. 103-127.

${ }^{8}$ Johnson, M., Littlejohn, D., Nazeer, W., Smith, K., and Cheng, R., "A Comparison of the Flowfields and Emissions of High-Swirl Injectors and Low-Swirl Injectors for Lean Premixed Gas Turbines," Proceedings of the Combustion Institute, Vol. 30, 2005, pp. 2867-2874.

${ }^{9} \mathrm{Li}$, G. and Gutmark, E., "Effect of Exhaust Nozzle Geometry on Combustor Flow Field and Combustion Characteristics," Proceedings of the Combustion Institute, Vol. 30, 2005, pp. 2893-2901.

${ }^{10} \mathrm{Fu}$, Y., Cai, J., Jeng, S.-M., and Mongia, H., "Confinement Effects on the Swirling Flow of a Counter-Rotating Swirl Cup," ASME Turbo Expo, 2005.

${ }^{11} \mathrm{Ji}$, J. and Gore, J., "Flow Structure in Lean Premixed Swirling Combustion," Proceedings of the Combustion Institute, Vol. 29, 2002, pp. 861-867.

${ }^{12}$ Mongia, H. C., "TAPS - A 4th Generation Propulsion Combustor Technology for Low Emissions," AIAA Paper, 2003, pp. 2003-2657.

${ }^{13}$ Stouffer, S., Ballal, D., Zelina, J., Shouse, D., Hancock, R., and Mongia, H., "Development and Combustion Performance of a High Pressure WSR and TAPS Combustor," AIAA Paper, 2005, pp. 2005-1416.

${ }^{14}$ Menon, S. and Patel, N., "Subgrid Modeling for Simulation of Spray Combustion in Large-Scale Combustors," $A I A A$ Journal, Vol. 44, 2006, pp. 709-723.

${ }^{15}$ Clemens, N. and Mungal, M., "A planar Mie scattering technique for visualizing supersonic mixing flows." Experiments in Fluids, Vol. 11, 1991, pp. 175-185.

${ }^{16}$ Samimy, M. and Lele, S., "Motion of particles with inertia in a compressible free shear layer." Physics of Fluids A, Vol. 3, 1991, pp. 1915-1923.

${ }^{17}$ Vandooren, J., deGuertechin, L., and van Tiggelen, P., "Kinetics in a Lean Formaldehyde Flame," Combustion and Flame, Vol. 64, 1986, pp. 127-139.

${ }^{18}$ Smooke, M., Mitchell, R., and Keyes, D., "Numerical Solution of Two-Dimensional Axisymmetric Laminar Diffusion Flames," Combustion Science and Technology, Vol. 67, 1989, pp. 85-122.

${ }^{19}$ Peeters, J. and Mahnen, G., "Reaction Mechanisms and Rate-Constants of Elementary Steps in Methane-Oxygen Flames," Proceedings of the Combustion Institute, Vol. 14, 1973, pp. 133-146.

${ }^{20}$ Harrington, J. and Smyth, K., "Laser-Induced Fluorescence Measurements of Formaldehyde in a Methane/Air Diffusion Flame," Chemical Physics Letters, Vol. 202, 1993, pp. 196-202.

${ }^{21}$ Brackmann, C., Nygren, J., Bai, X., Li, Z., Bladh, H., Axelsson, B., Denbratt, I., Koopmans, L., Bengtsson, P., and Aldén, M., "Laser-Induced Fluorescence of Formaldehyde in Combustion Using Third Harmonic Nd:YAG Laser Excitation," Spectrochimica Acta, Part A: Molecular and Biomolecular Spectroscopy, Vol. 59, 2003, pp. 3347-3356. 
${ }^{22}$ Rasmussen, C., Dhanuka, S., and Driscoll, J., "Visualization of Flameholding Mechanisms in a Supersonic Combustor Using PLIF," Proceedings of the Combustion Institute, Vol. 31, 2007, pp. 2505-2512.

${ }^{23}$ Bombach, R. and Käppeli, B., "Simultaneous Visualization of Transient Species in Flames by Planar-Laser-Induced Fluorescence Using a Single Laser System." Applied Physics B - Lasers and Optics, Vol. 68, 1999, pp. 251-255.

${ }^{24}$ Dhanuka, S., Temme, J., and Driscoll, J. F., "Vortex Shedding and Mixing Layer Effects on Periodic Flashback in a Lean Premixed Prevaporized Gas Turbine Combustor," To Appear: Proceedings of the Combustion Institute, Vol. 32, 2008. 